\title{
Role of Stress in Erectile Dysfunction: A Review
}

\author{
Dr. Akhil K Padmanabhan, MDS \\ Practitioner, Researcher \& Health advisor, India
}

*Corresponding Author: Dr. Akhil K Padmanabhan, MDS, Practitioner, Researcher \& Health advisor, India.

\begin{abstract}
Erectile dysfunction (ED) is a complex disorder that is studied to be of multifactorial in etiology. Although it commonly affects men above 50, there is also an increased incidence of ED observed in young men today. Several systemic disorders have been found to be linked to ED. Stress is a common disorder that affects a large population and is found to be a cause for ED in middle aged men. This article is an attempt to summarize the evidences in literature that studied the association between stress and ED and also the management if stress associated ED.
\end{abstract}

Keywords: Erectile dysfunction, Stress, Inflammation, Sexual disorders.

\section{INTRODUCTION}

Stress is a common disorder in the present day life. Prolonged stress can have a detrimental effect on the systemic health of the individual. This includes immunosuppression, loss of libido, cardiovascular diseases and inflammatory diseases. [1] In men, stress is shown to potentially cause sexual disorders of which Erectile Dysfunction (ED) is of greater significance.

Erectile dysfunction is a common sexual disorder which is characterized by the disability to get an erection during sexual activity. [2] It is more common in men above 40 years of age. [3] There have been several factors that are studied to be causing ED such as neurogenic, cavernosal, psychological, hormonal and drugs, [4] however, the most commonly explained pathophysiology is vascular disease. [5]

\subsection{Pathogenesis of Erectile Dysfunction}

Followed by sexual stimulation, there is a relaxation of smooth muscles and vasodilatation in the penis. This is mediated my certain neurotransmitters in the brain and also by mediators such as Nitric Oxide (NO) and Cyclic GMP (cGMP). A downregulation of these mediators at any level or the alterations in the autonomic activity can lead to failure in attaining an erection leading to ED. [6]

\subsection{Mechanisms by which Stress can Cause ED}

Stress can cause release of cateholamines which on a prolonged period can cause hypertention. [7] Erectile competence may be affected in multiple ways by the variations in autonomic activity basally present in hypertension, such as an increased sympathetic activity. This neurophysiological alteration can lead to several sexual disorder including premature ejaculation and ED and is considered one of the most attributable cause in the development of ED in stressed individuals or the worsening of already existing ED. [8]

Stress can also cause an increase in the systemic inflammation. This essentially involves release of certain chemical mediators including interleukins. Also, stress can lead to increased steroid levels and certain vasoconstrictors. All of these changes collectively can lead to endothelial dysfunction. [9]

Furthermore, mental stress can cause a dysregulation of circulatory mechanisms in both acute and chronic phases. Vascular disorders are considered to be the main etiological factor in development of ED. It can potentially impair the blood flow to the penis during sexual stimulation which can lead to an inability to attain and maintain an erection.

An increase in the oxidative stress can decrease the chemical mediators such as NO which is 
essential for erection to happen. This is also considered a mechanism by which prolonged mental stress can cause ED. [9] Individuals with existing ED can have stress due to poor sexual performance which can further contribute to stress thus leading to worsening of the condition (Figure 1).

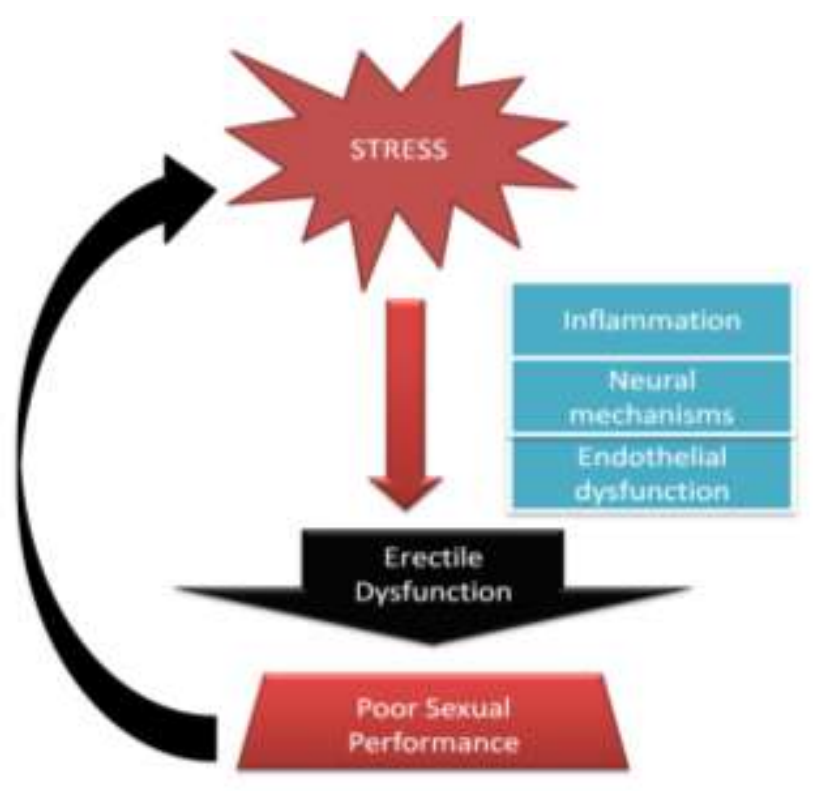

Figure1. The diagrammatic representation of stress causing ED

\section{CONCLUSION}

ED is a complex disorder with several factors playing a role. The hemodynamic and neurological changes caused by mental stress can contribute to ED. For the successful management of ED, examination for stress and other lifestyle diseases should be performed as a part of the initial diagnosis. Management of stress can be beneficial in treating ED. Furthermore, the still largely unexplored interaction mental stress and sexual disorders requires further investigations and large scale clinical trials.

\section{REFERENCES}

[1] Rice PL. Stress and health. Pacific Grove, CA: Brooks/Cole Publishing; 1999.

[2] Montague DK, Jarow JP, Broderick GA, Dmochowski RR, Heaton JP, Lue TF, et al. Chapter 1: The management of erectile dysfunction: An AUA update. J Urol 2005; $174: 230-9$

[3] Krane RJ, Goldstein I, Saenz de Tejada I. Impotence. N Engl J Med 1989;321:1648-59.
[4] Chiurlia E, D'Amico R, Ratti C, Granata AR, Romagnoli R, Modena MG. Subclinical coronary artery atherosclerosis in patients with erectile dysfunction. J Am Coll Cardiol 2005;46:1503-6

[5] Heidelbaugh JJ. Management of erectile dysfunction. Am Fam Physician 2010;81:305-12

[6] De Tejada IS, Angulo J, Cellek S, González-Cadavid N, Heaton J, Pickard R, Simonsen U. Pathophysiology of erectile dysfunction. The journal of sexual medicine. 2005 Jan 1;2(1):26-39.

[7] Kulkarni S, O'Farrell I, Erasi M, Kochar MS. Stress and hypertension. WMJ: official publication of the State Medical Society of Wisconsin. 1998 Dec;97(11):34.

[8] Pagani M. Hypertension, stress and erectile dysfunction: potential insights from the analysis of heart rate variability. Current medical research and opinion. 2000 Jan 1;16(sup1):s3-8.

[9] Schulz E, Jansen T, Wenzel P, Daiber A, Muenzel T. Nitric oxide, tetrahydrobiopterin, oxidative stress, and endothelial dysfunction in hypertension. Antioxidants \& redox signaling. 2008 Jun 1;10(6):1115-26.

Citation: Dr. Akhil K Padmanabhan, MDS. Role of Stress in Erectile Dysfunction: A Review. ARC Journal of Urology. 2020; 5(1):1-2. DOI: https://doi.org/ 10.20431/2456-060X.0501001.

Copyright: () 2020 Authors. This is an open-access article distributed under the terms of the Creative Commons Attribution License, which permits unrestricted use, distribution, and reproduction in any medium, provided the original author and source are credited. 